\title{
Le couple État-territoire en droit international contemporain
}

\section{Maurice Flory}

\section{(2) OpenEdition}

1 Journals

Édition électronique

URL : http://journals.openedition.org/conflits/255

DOI : 10.4000/conflits.255

ISSN : 1777-5345

Éditeur :

CCLS - Centre d'études sur les conflits lilberté et sécurité, L'Harmattan

Édition imprimée

Date de publication : 15 mai 1996

ISSN : 1157-996X

Référence électronique

Maurice Flory, «Le couple État-territoire en droit international contemporain », Cultures \& Conflits [En

ligne], 21-22 | printemps-été 1996, mis en ligne le 15 mars 2006, consulté le 30 mars 2021. URL

http://journals.openedition.org/conflits/255 ; DOI : https://doi.org/10.4000/conflits.255

Ce document a été généré automatiquement le 30 mars 2021.

Creative Commons License 


\title{
Le couple État-territoire en droit international contemporain
}

\author{
Maurice Flory
}

1 Le droit international a sans doute sa place dans une recherche sur le thème de “ l'International sans territoire ", encore que le territoire observé par le juriste soit bien différent de celui du politiste. Conscient de toutes les transformations intervenues au cours des quatre ou cinq dernières décennies, le juriste ne conteste pas les observations pertinentes et les explications fournies par la science politique sur la crise de l'État et par extension sur les problèmes posés au territoire. Pourtant son approche reste différente car il n'est pas seulement un observateur, il est aussi préoccupé du mode de gestion d'un espace qui n'est pas celui de l'État considéré pour lui-même, mais bien celui des espaces étatiques englobés dans une société mondiale. Un premier constat s'impose : pour le juriste de droit international, État et territoire sont étroitement liés et représentent ensemble l'élément fondateur de la société internationale. Le juriste n'oublie pas la finalité de sa discipline: depuis quelques trois siècles la société internationale repose sur l'État, un État qui prend un sens particulier au plan des relations internationales. Alors que le regard du politiste posé sur la structure interne de l'État s'intéresse aux particularités de chaque société, l'analyse de l'internationaliste cherche à réduire ces différences et à obtenir un commun dénominateur à partir duquel il est possible d'organiser la société internationale. De là vient cette égale souveraineté attribuée aux États en droit international. Cette formule indéfiniment répétée dans tous les grands textes du corpus juridique ${ }^{1}$ parait de moins en moins conforme à la réalité et pourtant cette affirmation a retrouvé toute son actualité avec les nouveaux États décolonisés; difficile à justifier à partir d'une analyse interne, elle trouve tout son sens dans une société internationale où la souveraineté de l'État est nécessairement limitée par celle des autres, ce qui impose l'égalité dans la souveraineté. L'État en droit international se définit alors comme une entité abstraite, dématérialisée par l'alchimie d'une fiction juridique; il se présente comme une notion fonctionnelle insensible aux dégradations. Égal et souverain par hypothèse, cet État va tirer de son caractère abstrait son utilité pratique puisque c'est sur ce postulat que va naître le droit international tel qu'il fonctionne aujourd'hui. Parce que le territoire est 
en droit international l'un des éléments constitutifs de cet État abstrait, il participe du même caractère. Il est l'espace de souveraineté dont dispose à titre exclusif tout État. De la confrontation entre égales souverainetés résulte la nécessité d'une délimitation qui fait apparaître la frontière destinée à empêcher tout empiétement sur une autre souveraineté. L'organisation globale des espaces géographiques à partir de territoires souverains engendre une société d'États juridiquement homogène. L'idée récemment réapparue de terrae incognitae qui suggère une vacance de pouvoir sur certains territoires n'a pas place dans cette société internationale idéalisée où tout espace relève nécessairement de l'un de ces États également souverains. Ces considérations mettent en lumière le décalage existant entre la science politique et le droit. Le texte de présentation de cette recherche déclare fermement: "A scène nouvelle, sociologie des relations internationale renouvelée ". Ce qui est imaginable en sociologie ne l'est pas aussi facilement en droit. Le droit assure la continuité ; il doit être opérationnel ; avant de dénoncer les faiblesses d'un concept, il s'interroge sur les alternatives possibles. Le titre de la présente communication réunit le territoire à l'État sous la forme d'un couple, c'est à dire d'un ensemble de deux éléments indissociables. En droit international, on ne peut en effet concevoir l'un sans l'autre car un État suppose un territoire et parce qu'en dehors des territoires antarctiques, il n'existe pas de territoire sans État. En quelques rares exceptions, l'abstraction de ces concepts a pu permettre des partages de souveraineté ou un exercice extraterritorial limité de la souveraineté. Mais ces exceptions n'ont pas ébranlé les bases de l'État territorial, pas plus que ce qu'on appelle aujourd'hui la crise de l'État ne parait remettre en cause les fondements du Droit international. " International sans territoire ", est imaginable en science politique, mais le droit international d'aujourd'hui ne se conçoit pas sans territoire. A une détérioration apparente du concept classique du territoire, répond sa pérennité juridique.

2 Les apparences d'une détérioration du territoire Le titre juridique essentiel de la compétence étatique est la souveraineté territoriale. L'exclusivité de juridiction sur son territoire est un postulat appliqué à tout État. La jurisprudence internationale l'a rappelé chaque fois qu'à l'occasion d'une contestation, le juge a dû déterminer quel était le titulaire de la souveraineté. Les cas de partage de la souveraineté n'ont existé que sous la pression de la colonisation, dans les régimes de Protectorat ${ }^{2}$, ou imposés par la guerre à travers l'installation autoritaire de gouvernements satellites dépendants, ou encore comme conséquence de l'occupation militaire qui ne dispose d'après le droit de la guerre que d'une compétence limitée ${ }^{3}$. Mais les situations de ce type sont passagères ${ }^{4}$, comme le sont les différends territoriaux qui finissent par trouver une solution. Ce qui aujourd'hui rend le territoire fragile semble venir d'un malaise identitaire plus profond.

Les contestations du territoire Les conflits territoriaux ont toujours existé. La France considérée comme un Etat-nation type particulièrement stable, n'en a pas moins connu jusqu'en 1945 de nombreuses modifications territoriales et rectifications de frontières ${ }^{5}$. Ce n'est pas là qu'il faut chercher l'inquiétude actuelle mais du côté des États nouveaux qui n'existaient pas avant la colonisation; l'expression d'État importé ${ }^{6}$ indique bien leur fragilité. Il ne faudrait pas en déduire comme on l'a fait au XIXe siècle que le colonisateur n'avait trouvé à son arrivée qu'une terra nullius. La Cour internationale de Justice dans son avis sur la Sahara occidental a montré qu'existaient alors d'autres formes d'organisation du pouvoir sans qu'il s'agisse stricto sensu d'État ${ }^{7}$. Pourtant le 
plus souvent, les autorités coloniales ont transposé leurs méthodes, à commencer par les exigences de la souveraineté territoriale. Il était indispensable de savoir jusqu'où s'étendait le pouvoir souverain ; il fallait délimiter l'espace colonisé. L'établissement de cette frontière fut négocié avec le souverain voisin qui était le colonisateur britannique, italien, portugais ou belge, ou encore discuté avec le collègue administrateur colonial de la circonscription voisine en fonction des nécessités d'une bonne gestion administrative. Le caractère artificiel et importé de ces frontières coloniales a été fréquemment souligné et dénoncé. Il est vrai que c'est souvent au nom d'intérêts étrangers aux habitants du pays que se sont opérées ces divisions. Mais ces critiques doivent être relativisées, car l'établissement d'une administration moderne qui supposait des limites précises n'a pas eu que des inconvénients. Il est d'ailleurs frappant de constater qu'une des premières résolutions votées à l'Organisation de l'Unité africaine par ses États membres a été le ralliement à l'uti possidetis, c'est à dire au statu quo territorial laissé par le colonisateur ${ }^{8}$. Décision sage à un moment où ces nouveaux États avaient tant à faire pour se mettre en place. Mais il n'est pas étonnant que par la suite soient apparues des malformations territoriales, des espaces qui, compte tenu de la géographie ou des ethnies, auraient dû connaître d'autres découpages. Un abondant contentieux territorial s'en est inévitablement suivi. Le côté artificiel de cette délimitation géographique n'est pas apparu seulement par l'existence de ces frontières nouvelles. Il s'est manifesté plus fondamentalement sur la signification même d'un espace territorial de souveraineté exclusive. Tant que les frontières sont restées plus théoriques que réelles, les populations ont pu conserver leurs habitudes peu attentives aux délimitations. Mais les autorités des nouveaux États ont naturellement manifesté un désir d'affirmer la souveraineté qu'ils venaient de conquérir et ont donc eu tendance à rigidifier la frontière, à lui donner une rigueur analogue à celle existant dans les pays développés. Il s'en est suivi des difficultés nées des contestations des États voisins et aussi de l'incompréhension des populations ellesmêmes ${ }^{9}$. La situation a pu s'aggraver lorsque deux ethnies traditionnellement hostiles, mais pacifiées par l'administration coloniale, se sont retrouvées après son départ seules face à face sur un même territoire. Partie constitutive de l'État, le territoire ne peut s'en dissocier, sous peine de retomber dans cette catégorie douteuse des territoires sans maitre ${ }^{10}$. Or la défaillance du pouvoir dans certaines régions peut atteindre un degré tel qu'il n'est plus possible de parler d'exercice de la souveraineté. La désintégration de l'État se répercute aussitôt sur le territoire livré à l'anarchie. Sans doute est-ce là une situation extrême qui n'a pas de nom dans le droit international contemporain ${ }^{11}$. Cette situation n'est pas propre à l'Afrique; on a fait remarquer qu'elle existe aussi dans certains territoires des pays de l'Est qui connaissent parfois les difficultés cumulées de la " décommunisation " et de la décolonisation. On en vient à se demander dans certains cas quel est le statut de ces territoires, qui les administre, où passent leurs frontières? Faut-il étendre cette inquiétude, comme certains ont cru pouvoir le faire, aux pays industrialisés qui connaissent eux aussi, notamment dans certains quartiers des grandes villes, une crise d'autorité et une carence de gestion? Sans doute y a-t-il là une situation tout à fait anormale dans un État développé et l'on comprend la crainte d'une extension du phénomène. Mais l'alerte étant donnée, ces États modernes et développés sont tout à fait en mesure d'exercer leur autorité sur les quelques rues d'un quartier où surgit ce type de phénomène. Il ne s'agit pas d'une remise en cause de la souveraineté territoriale comparable au désordre de la Somalie ou du Caucase. Cette pathologie constatée dans les grands centres urbains n'est pas la 
plus inquiétante. Elle suscite en effet, du moins dans les pays développés, les interrogations nécessaires à la recherche de remèdes adaptés. Il existe d'autres formes de détérioration plus subtiles et qui sans remettre en cause radicalement le territoire et avec lui l'État lui même, semblent cependant provoquer une mutation en profondeur qui annonce peut être de plus grands changements dans la société internationale. Les nouvelles solidarités trans-territoriales ${ }^{12}$ L'État souverain qui trouve son accomplissement dans l'État-nation apparaît dans le monde d'aujourd'hui souvent trop étroit. La mondialisation de l'économie, l'existence de superpuissances continentales obligent les États à s'organiser en se regroupant. Les Européens pour être à un niveau comparable à celui des États-Unis ou du Japon se sont unis dans une Communauté qui prend dans la vie même de ces États une place toujours plus importante. La méthode utilisée est celle du Fédéralisme qui suppose une décision prise en toute liberté et reste donc compatible avec la souveraineté. Mais les mécanismes du grand marché et maintenant de l'Union aboutissent à des transferts de compétence d'une importance telle que, dans certains secteurs, l'opinion publique ne rencontre plus la souveraineté là où traditionnellement on était habituée à la trouver. C'est ce qui existe déjà pour le commerce et ce qui se prépare pour la monnaie unique. Au territoire national se superpose alors un territoire de l'Union ce qui implique dans des secteurs unifiés la suppression des barrières qui séparaient les États membres. La seule frontière commerciale est désormais celle qui existe à l'entrée du territoire de la Communauté ; la conséquence visible en est la suppression programmée des postes douaniers intracommunautaires. Ce qui a été accepté sans difficulté pour les marchandises, l'est déjà moins pour la monnaie et encore moins pour les personnes; les règles touchant à l'accès au territoire national et à l'acquisition de la nationalité font partie des prérogatives essentielles de la souveraineté étatique. Le traité de Rome proclame pourtant la liberté de circulation des personnes. Mais dans le même temps chaque État entend rester maître de la politique d'accès à son territoire. Il va donc falloir gérer ces deux espaces dont l'un est communautaire et dont l'autre reste national. Ainsi s'expliquent les régimes différents applicables aux populations présentes sur le territoire de l'Union; la liberté de circulation des personnes, conséquence du grand marché, ne vaut que pour les ressortissants des États membres; pour les populations étrangères à l'Union, chaque État membre se réserve de faire ensuite les distinctions lui permettant de réglementer pour lui même l'accès à son territoire national, l'accès à l'emploi, la possibilité du regroupement familial, etc.... Seul le régime social de ces travailleurs étrangers est réglementé au niveau européen par l'obligation de non discrimination. Les deux espaces ne sont évidemment pas totalement indépendants l'un de l'autre. Mais comme les États membres n'ont pas donné compétence à l'Union en ce domaine, il leur revient de réaliser par des accords bi ou multilatéraux les aménagements qui leur paraissent nécessaires dans le domaine des contrôles et de la surveillance du territoire national ; cette préoccupation a conduit aux accords extracommunautaires de Schengen. Ainsi s'explique la complexité des règles de séjour qui font intervenir des textes communautaires, des conventions multilatérales et des lois nationales ${ }^{13}$. Le Fédéralisme est un processus évolutif. Pendant toute la période de décolonisation il a fonctionné dans un sens ségrégatif (Commonwealth, Union française, Communauté française) ; aujourd'hui on le voit agir dans un sens agrégatif, comme le montrent les diverses entreprises de régionalisation. L'expérience la plus avancée est celle de l'Europe. Bien que ralentie par la multiplication des parties prenantes, la construction européenne a déjà modifié sensiblement la portée du 
concept de territoire étatique, à tel point qu'on peut s'inquiéter de savoir si l'importance respective des territoires nationaux et du territoire de l'Union, n'est pas en train de s'inverser. Signes tangibles parmi d'autres : les passeports sont émis sous le double timbre des deux entités et dans les aéroports de l'Union pour les contrôles de police, les ressortissants de la Communauté bénéficient d'une file d'attente distincte. On ne peut exclure l'aboutissement d'une évolution difficile et sans doute lointaine par laquelle les États actuels renonceraient à leur souveraineté au bénéfice de l'Union devenue État fédéral. La souveraineté territoriale appartiendrait alors à l'Union, les États membres conservant un territoire et des compétences propres attribuées par la constitution de l'Union ${ }^{14}$. Mais d'autres phénomènes se manifestent déjà. Ainsi l'apparition d'une frontière économique englobant tout l'espace communautaire, suscite de nouvelles solidarités territoriales infra-nationales entre les États membres. Des régions appartenant à des États différents, mais géographiquement voisines ou économiquement complémentaires peuvent organiser des solidarités transfrontières directes, sans passer par le canal de leurs instances nationales respectives. De même la pratique des jumelages va créer des liens entre villes étrangères, non seulement au niveau des autorités municipales mais à celui de la société civile. Les relations interuniversitaires ont créé les outils nécessaires aux échanges d'étudiants et de professeurs; le succès des programmes ERASMUS en est une bonne illustration. Des Organisations non gouvernementales, en marge des États et sans la contrainte de la souveraineté, tendent à devenir des acteurs fort actifs de la vie internationale là où la frontière nationale constitue un obstacle à surmonter, dans les domaines humanitaires et écologiques notamment. L'ONU attribue à certaines d'entre elles une place officielle consultative dans ses instances économiques et sociales ${ }^{15}$. De la même façon, on sait l'importance des sociétés multinationales dont la puissance et la prospérité reposent précisément sur leur capacité à ignorer les traditionnelles frontières nationales, ce qui ne va pas sans poser à la souveraineté des États des problèmes de surveillance et de contrôle non résolus ${ }^{16}$. Ainsi se multiplient des réseaux transnationaux qui bouleversent les traditionnels clivages nationaux. La vie internationale n'est donc plus comme au début du siècle celle des seuls États souverains avec leur territoire strictement délimité par une frontière. La mondialisation de l'économie, les facilités de communication font qu'aujourd'hui la vie internationale se déroule à l'échelle du village mondial à la vie duquel sont appelés non seulement les États, mais aussi des institutions de plus en plus nombreuses à se considérer comme transnationales, des personnes toujours plus mobiles et des organisations internationales géographiquement très diverses et matériellement plus ou moins spécialisées. La notion de territoire national est désormais concurrencée par d'autres types d'espaces à dimension fédérative ou transnationale. Il existe une coexistence d'espaces territoriaux qui enlève au territoire national la force qui s'attachait à son caractère unique. Il est donc permis de se demander si le territoire national n'est pas en train de perdre progressivement sa raison d'être.

5 La pérennité juridique du territoire Les transformations observées viennent incontestablement changer le paysage juridique international. Pourtant si le territoire national se trouve en concurrence avec d'autres espaces territoriaux, c'est cependant bien lui qui continue à délimiter l'aire de souveraineté de l'État. État et territoire indissolublement liés quelles que soient les transformations, se consolident mutuellement et forment toujours la pierre angulaire du droit international. 
6 Les aménagements de la souveraineté territoriale Le titre " l'international sans territoire " fait référence au territoire étatique et non au territoire géographique qui est une donnée physique indestructible. Mais dès lors qu'un espace géographique est administré, il renvoie à une compétence qui suppose une limite spatiale. Traditionnellement, l'État souverain organisait à sa convenance l'administration de l'espace qu'il contrôlait. Désormais il lui faut tenir compte d'autres espaces de compétence sur son propre territoire. Inversement, pour des raisons de troubles et de sous développement, des États peuvent cesser de contrôler la totalité de leur territoire ; la Communauté internationale se trouve alors confrontée à un problème de vacance de pouvoir qu'elle ne peut ignorer. Dans le premier cas, c'est la technique fédérale qui fournit la solution. Le Fédéralisme permet en effet de faire coexister harmonieusement plusieurs étages décisionnels. Cela suppose que soit fixé à l'origine le niveau de la compétence fondatrice, la compétence-mère qui initiera toutes les autres. Or à cet égard, aucun doute n'est possible et aucun changement n'a modifié le principe de base ; cette compétence initiale reste toujours celle de l'État précisément qualifié pour cette raison de souverain. C'est donc à cet État qu'il revient d'organiser la répartition des compétences au dessous et au dessus de lui. Au dessous, il agira en toute liberté et aura le choix entre l'adoption d'une structure fédérale ou d'une gestion plus ou moins décentralisée. Au dessus il lui sera possible d'entrer dans un système fédéral négocié à égalité avec ses partenaires souverains par un traité international qui devra répartir les niveaux de compétence entre ce que les autorités de l'État veulent conserver et ce qu'elles acceptent de transférer au niveau fédéral. La méthode fédérale présente l'avantage d'être respectueuse des compétences de chacun tout en restant souple puisqu'elle permet par des mécanismes adaptés tel que le dédoublement fonctionnel si bien analysé par G. Scelle ${ }^{17}$, de permettre à un ordre juridique trop faible d'emprunter à un autre ordre les instruments qui lui font défaut sans que pour autant il y ait confusion des compétences. Le cas d'une vacance de souveraineté sur certains territoires pose à la communauté internationale un problème inédit. Au temps de la colonisation, une terre qui n'était pas rattachée à un État était qualifiée de res nullius, ce qui la rendait colonisable selon les moeurs de l'époque. Les cas de déshérence qui se présentent aujourd'hui appartiennent à une autre époque, mais surtout ils sont d'une nature différente puisqu'il s'agit d'un territoire relevant d'un État internationalement reconnu, mais qui en raison de l'anarchie régnante n'est plus contrôlé par son administration. Ce territoire dont le rattachement à un État n'est plus que fictif, échappe par là-même aux règles de la vie internationale ; il peut alors devenir le siège de toutes les violations du droit et mettre en péril la paix et les droits de l'homme. Devant une telle situation, il est difficile à la communauté internationale, dès lors qu'elle est informée de violations graves du droit, de ne pas réagir. Mais elle ne peut plus le faire en mettant en cause son interlocuteur institutionnel habituel qu'est l'État incapable en l'occurrence d'administrer son territoire. Pourtant ce dernier, n'ayant pas renoncé à sa souveraineté territoriale, n'acceptera pas une ingérence qu'il considérera comme contraire à l'art. $2 \S 7$ de la Charte des Nations Unies. La fiction de l'État souverain sur son territoire bloque toute intervention extérieure. Plusieurs tentatives ont été faites ces dernières années pour surmonter cet obstacle. La première est celle de l'Assemblée générale des Nations-Unies. par sa résolution 43/131 du 8/12/1988 sur “ l'assistance humanitaire aux victimes des catastrophes naturelles et situations d'urgence du même ordre ". Après avoir réaffirmé, comme il se doit, la souveraineté des États, la résolution invite les États qui ont besoin d'une telle assistance à faciliter la 
mise en oeuvre de l'aide humanitaire. Une seconde résolution du 14/12/1990 se fondant sur l'expérience, tente de réduire l'impact trop large de l'activité humanitaire sur la souveraineté territoriale en suggérant " la possibilité de créer à titre temporaire, là où il est nécessaire et de manière concertée entre les gouvernements touchés et organisations intergouvernementales, gouvernementales et non gouvernementales intéressées, des couloirs d'urgence pour la distribution d'aide médicale et alimentaire d'urgence ". Mais ces deux textes ne sont que des recommandations non obligatoires; leur application reste à la discrétion de l'État qui peut toujours leur opposer l'exclusivité de sa souveraineté territoriale ${ }^{18}$. Une autre voie pour l'aide humanitaire est celle des Organisations non gouvernementales; ne se réclamant d'aucune prérogative de souveraineté, elles peuvent apparaître plus facilement acceptables pour l'État hôte qu'une assistance étatique. Il n'en reste pas moins que le souverain est censé rester maître de l'accès sur son territoire et que les ONG doivent donc travailler sur ce territoire munies d'une autorisation. A défaut, les membres de ces ONG interviennent à leurs risques et périls. Il reste enfin la possibilité d'utiliser la seule exception à la souveraineté territoriale prévue par la Charte, celle du Chap.VII, lorsqu'il y a menace contre la paix. Par une interprétation extensive du concept de menace à la paix, le Conseil de sécurité a voté à plusieurs reprises des interventions humanitaires réalisées directement par des opérations de maintien de la paix sous son contrôle direct, ou opérées indirectement par délégation aux forces armées d'un État membre ${ }^{19}{ }^{20}$. Le résultat de l'opération ne sera pas toujours à la hauteur des espérances; pour qu'il soit durable il conviendrait de replacer le territoire en vacance de pouvoir sous l'autorité retrouvée de l'État, autrement dit de restaurer l'État dans son intégrité territoriale. Qui peut aider à cette restauration? L'ONU ne dispose pas d'institutions pour administrer ou faire administrer un territoire puisque en vertu de l'art.78 de la Charte "le régime de tutelle ne s'appliquera pas aux pays devenus membres des $\mathrm{NU}$, les relations entre celles-ci devant être fondées sur le respect du principe de l'égalité souveraine ". Pourtant l'ONU en quelques occasions a assumé une gestion provisoire, notamment dans une étape de décolonisation en Irian occidental ${ }^{21}$ et en $\mathrm{Namibie}^{22}$. Plus récemment dans le cas du Cambodge ${ }^{23}$ et de la Somalie ${ }^{24}$, c'est bien une restauration de l'État qui lui fut demandée par le moyen de vastes opérations de maintien de la paix. Si elle a fait des efforts tout à fait remarquables ces dernières années, l'ONU n'a pu réaliser pleinement ses ambitieux programmes, car elle n'avait pas reçu de la Charte les moyens et les compétences nécessaires pour administrer un territoire et reconstruire un État. Elle peut sous différentes formes et notamment en utilisant ce qu'on a appelé la deuxième génération des opérations de maintien de la paix, apporter une coopération dans un certain nombre de domaines; mais elle ne dispose pas des instruments réservés à la souveraineté et qui restent l'apanage du seul État. La souveraineté territoriale continue à être dans le monde actuel une institution irremplaçable, comme ne cesse de le confirmer la jurisprudence internationale.

7 La souveraineté territoriale dans la jurisprudence internationale De nombreux litiges ont permis aux juges et aux arbitres de préciser les caractères et le rôle fondamental du territoire. Ils ont d'abord rappelé le sens de la plénitude et de l'exclusivité de la souveraineté territoriale, puis ont confirmé pour en assurer le plein exercice la nécessité d'une définition sans ambiguïté de ses limites géographiques. Cette souveraineté est généralement affirmée sous son angle politique, mais il faudra encore préciser le sens de l'expression souveraineté économique couramment utilisée dans la terminologie des Nations unies à partir des années soixante. C'est la sentence rendue 
par Max Huber dans le cadre de la Cour Permanente d'Arbitrage en 1928 qui fixe les principes de base de la souveraineté territoriale: "La souveraineté dans les relations entre États signifie l'indépendance. L'indépendance relativement à une partie du globe est le droit d'y exercer, à l'exclusion de tout autre État les fonctions étatiques ... ". L'affaire du détroit de Corfou (CIJ 1949) est venue confirmer " qu'entre États indépendants, le respect de la souveraineté territoriale est une des bases essentielles des rapports internationaux ". La jurisprudence n'a pu que confirmer l'illicéité de tout acte de contrainte en territoire étranger (Affaire du personnel diplomatique américain, CIJ 1980) Cette exclusivité de compétence supposant une délimitation territoriale stricte, la jurisprudence est en ce domaine particulièrement fournie pour les frontières terrestres et plus encore pour les limites en mer compte tenu de la convention de Montego Bay qui a nécessité de nouvelles délimitations. En ce qui concerne les premières, l'effectivité reste l'élément décisif de toute affectation de territoire; puisque le territoire est l'espace exclusif de souveraineté, il s'agit de vérifier, en cas de concurrence, qui exerce la souveraineté avec le plus de continuité (Affaire des Minquiers et des Ecrehous, CIJ 1953 ; Avis consultatif sur le Sahara occidental, CIJ 1975). Le contentieux des espaces maritimes est de nature différente dans la mesure où la réponse relève non pas de l'effectivité, mais de règles de délimitation inscrites dans les conventions souvent difficiles à appliquer concrètement ${ }^{25}$. La souveraineté n'a de sens que prise dans sa plénitude. Elle englobe tous les éléments nécessaires de la vie de l'État. Pourtant il a paru nécessaire de préciser qu'elle comportait un aspect économique en raison de la colonisation qui a souvent débuté par l'aliénation d'éléments importants de l'économie. Durant la période coloniale des investisseurs étrangers se sont intéressés à l'exploitation des richesses minières et pétrolières. Ils ont implanté des sociétés qui sont devenues prospères. Une fois indépendants, les nouveaux États ont estimé que pour maitriser l'économie de leur pays, il leur fallait contrôler l'exploitation de ces richesses et donc nationaliser. D'où le principe de " souveraineté permanente sur les ressources naturelles et les activités économiques " maintes fois proclamée dans les résolutions de $\mathrm{l}^{\prime} \mathrm{ONU}^{26}$. La jurisprudence internationale saisie de plusieurs affaires importantes a tranché en faveur de la souveraineté territoriale, mais dans le respect des obligations contractuelles, à condition que cellesci ne soient pas contraires à la souveraineté27. Ainsi se confirme le fait que lorsque surgit un différend relatif à un problème de souveraineté territoriale, c'est toujours en faveur de la consolidation et du respect de la souveraineté que vont trancher l'arbitre ou le juge. Comment en effet pourraient-ils statuer différemment sans mettre en cause l'État lui-même sur lequel repose toute la construction du Droit international ? En conclusion, la notion de territoire en droit international n'est pas en crise. Sans doute les différends existent-ils; ils vont parfois jusqu'à l'affrontement ouvert et ils n'ont peut être jamais été aussi nombreux ; mais le Droit international dispose de procédures qui ne cessent de rappeler les principes sur la base desquels la solution doit être trouvée. Les constructions fédérales, si elles bouleversent des habitudes, ne constituent aucunement une atteinte au concept juridique de territoire. Sans doute les phénomènes de trans-territorialité méritent-ils de retenir toute notre attention; pourtant s'ils compliquent la situation, ils n'effacent pas le rôle de répartiteur des compétences que joue le territoire ; les entités transnationales n'ignorent pas le territoire, comme on le dit parfois ; elles ont simplement l'avantage de pouvoir jouer sur plusieurs d'entre eux. Si le territoire conserve une place irremplaçable en droit international, c'est bien parce qu'il n'existe pour l'instant aucune alternative. La vision marxiste d'un monde unifié où 
l'État dépérirait pour disparaitre a rejoint dans l'histoire des idées bien d'autres utopies. L'Islam rassemblant dans une même foi les hommes sur un Dar El Islam unique relève d'un mythe qui puise ses origines dans l'histoire reconstruite et extrapolée des quatre premiers califes à l'origine de l'expansion arabe ${ }^{28}$. Quant à l'avènement d'un monde géré par les multinationales qui auraient fini par se substituer aux États, on ne les trouve que dans les romans ou les films d'anticipation. Le territoire, espace de souveraineté de l'État, reste donc toujours l'élément fondamental de la construction et du fonctionnement de la société internationale. On peut ici reprendre la conclusion de Jean Pierre Queneudec au colloque de la Société française pour le droit international sur l'État souverain à l'aube du XXIe siècle en l'appliquant au territoire en tant que composante de l'État: “ Oui l'État existe en tant que catégorie juridique du droit international ; la notion présente une irréductible unité et sa pérennité parait assurée à l'aube du XXIe siècle " ${ }^{29}$.

\section{NOTES}

1. A commencer par la Charte des Nations Unies : art.2 $\$ 1$ “ l'Organisation est fondée sur le principe de l'égalité souveraine de tous ses membres..."

2. Maurice Flory, La notion de protectorat, LGDJ, 1955.

3. Odile Debbasch, L'occupation militaire, Pouvoirs reconnus aux forces armées hors de leur territoire national, LGDJ, 1962, notamment p. 245 et sq.

4. Les situations territoriales conflictuelles qui durent tiennent précisément au fait que l'établissement du titulaire de la souveraineté n'a pu être résolu ; le cas de la Palestine en est un exemple puisque le même territoire fait l'objet de revendications de la part de deux populations qui invoquent l'une et l'autre des titres indéniables.

5. Cf. C. Rousseau, Droit international public, T.III, Chap.3, “ Les frontières de la France ", Sirey 1977, p. 289.

6. B. Badie, L'Etat importé, Fayard, 1992. Voir aussi P-R. Baduel, États, nation et nationalisme, REMM n ${ }^{\circ}$ 68-69, p. 11 et P-R Baduel (dir.) États, territoires et terroirs au Maghreb, CRESM, CNRS, 1985

7. M. Flory, “l'Avis de la CIJ sur le Sahara occidental ”, AFDI, 1975, p. 272 : “ de l'avis de la Cour, aucune règle de droit international n'exige que l'Etat ait une structure déterminée...".

8. Résolution 16-1 de l'OUA, Le Caire, juillet 1964 : principe qui s'applique non seulement aux limites à l'intérieur des anciens empires coloniaux, mais encore à leurs limites extérieures (cf. la sentence arbitrale du 31/7/1989 sur la détermination de la frontière maritime Guinée-Bissau/Sénégal, RGDIP, 1990, p. 251).

9. Cf. par exemple les différents contentieux nés dans la zone sahélienne entre tous les pays qui entourent les terres de nomadisation du Sahara.

10. Voir l'Avis de CIJ sur le Sahara occidental, réponse à la première question, op. cit. 11. D'où le nom de terrae incognitae, exhumé des vieux atlas, que leur donne J.C. Rufin, L'empire et les nouveaux barbares, Paris, J.C. Lattès, Pluriel, 1991. 
12. Voir en parallèle à ce développement, dans l'ouvrage de B. Badie et de M.C. Smouts, Le retournement du monde, PFNSP et Dalloz, 1992, l'excellent chapitre 2 consacré à la montée des flux transnationaux, qui montre les différences de préoccupation du Droit et de la Science politique.

13. M.P. Lanfranchi, Droit communautaire et travailleurs migrants des Etats Tiers, Economica, 1994.

14. Sur cette évolution potentielle, la référence à la naissance des Etats Unis d'Amérique et à l'adoption de la Constitution fédérale est très instructive. Voir sur ce sujet, Bernard E. Brown, L'Etat et la politique aux Etats Unis, PUF, coll. Thémis, 1994, notamment pp. 5-85.

15. Art.71 de la Charte des NU : “Le Conseil économique et social peut prendre toutes dispositions utiles pour consulter les Organisations non gouvernementales qui s'occupent de questions relevant de sa compétence... ".

16. Le problème posé par les sociétés multinationales est évoqué en 1974 dans le Déclaration relative à l'instauration d'un Nouvel Ordre Economique International, AG 3201 S IV et dans la Charte des droits et devoirs economique des États AG 3281 XXIX. Le Programme d'action relatif à l'instauration d'un NOEI AG 3202 S IV de 1974 leur consacre son chap. V qui évoque pour la première fois l'idée d'un Code de conduite qui n'a jamais dépassé le stade de projet.

17. Voir notamment, G. Scelle, Précis de droit des gens, Sirey, 1934, II, p. 10.

18. Voir entre beaucoup d'autres, Mario Bettati, “ Un droit d'ingérence ”, RGDIP, 1991, p. 639.

19. La résolution $S .688$ du 5 avril 1991, après avoir rappelé l'art.2\$7 de la Charte, n'en impose pas moins l'accès immédiat des organisations humanitaires sur le sol irakien, en prenant la précaution d'employer la formule " insiste pour que l'Irak permette un accès immédiat ". La résolution S.794 du 4 décembre 1992 invoque le chap.VII pour autoriser l'intervention d'une force américaine (Restore Hope) destinée à permettre la présence d'une aide humanitaire. C'est aussi le chap.VII qui est invoqué pour l'aide humanitaire envoyée sous l'égide de la FORPRONU en Yougoslavie (S.724, 752, 757, 760, 770, 771, 775).

20. Autorité exécutive temporaire des NU (AETNU), Oct.1962-Mai 1963.

21. Autorité exécutive temporaire des NU (AETNU), Oct.1962-Mai 1963.

22. Groupe d'assistance des NU pour la période de transition (GANUPT) 1978-1990. L'element civil du GANUPT a joué une rôle administratif essentiel pour le contrôle des forces de police et l'organisation des élections, cf. M. Kamto, “L'accession de la Namibie à l'indépendance ", RGDIP, 1990, pp. 578-634.

23. Autorité provisoire des NU au Cambodge, (APRONUC) à partir de 1991, notamment CS 792 du 30/11/92, 810 du 8/3/93, 826 du 20/5/93, 880 du 4/11/93.

24. ONUSOM II, notamment CS $897 \mathrm{du} 3 / 2 / 94$

25. Cf. Prosper Weil, Perspectives du droit de la délimitation maritime, Pedone, 1988.

26. Notamment AG 2626 XXV, art. 74 (24 octobre 1970), AG 3201 et 3202 SIV (1 mai 1974), AG 3281 XXIX (12 décembre 1974).

27. Arbitrage Texaco du 17/1/1977, JDI, 1977, p. 374 ; Aminoil du 24/3/1982, JDI, 1982, p. 869.

28. Si la référence au Dar el Islam unifié est présente, au moins sous une forme allusive, dans beaucoup de discours politiques, elle ne s'est jamais concrétisée en dépit des occasions qui se présentaient. Cf. M. Flory, P.Agate (dir.) Le système régional arabe, 
CRESM, CNRS, 1989 ; voir aussi M.Flory, “ Régimes arabes et environnement international ", in Les régimes arabes, PUF, coll. Thémis, 1990.

29. Société française pour le droit international, Colloque de Nancy, L'État souverain à l'aube du XXIe siècle, Pedone 1994, p. 308.

INDEX

Mots-clés : droit international, Etat-nation, territoire(s) et territorialité, souveraineté 